\title{
Genome Sequence Resource of Phytophthora vignae, the Causal Agent of Stem and Root Rot of Cowpea
}

\author{
Chengdong Yang, ${ }^{1}$ Lianyu Lin, ${ }^{2}$ Jiandong Bao, ${ }^{2}$ Zhixin Wang, ${ }^{1}$ Zhiting Li, ${ }^{1}$ Hengyuan Guo, ${ }^{1}$ \\ Lin Lv, ${ }^{1}$ Dan $\mathrm{Yu},{ }^{1}$ and Qinghe Chen ${ }^{1, \dagger}$ \\ ${ }^{1}$ Key Laboratory of Green Prevention and Control of Tropical Plant Diseases and Pests, Ministry of \\ Education, College of Plant Protection, Hainan University, Haikou 570228, China \\ ${ }^{2}$ Fujian University Key Laboratory for Plant-Microbe Interaction, College of Life Sciences, Fujian \\ Agriculture and Forestry University, Fuzhou 350002, China
}

\section{Funding}

This work was supported by grants from the National Natural Science Foundation of China (31772141), and the Scientific Research Foundation of Hainan University [KYQD(ZR)-20080].

\begin{abstract}
The causal agent of stem and root rot of cowpea, Phytophthora vignae, is a widely distributed species of the Phytophthora genus. Here, we generate a high-quality complete genome assembly of $P$. vignae PSY2020 (89.39 Mb, $N_{50} 2.99 \mathrm{Mb}$ ) from China, using Oxford Nanopore Technologies (ONT) sequencing. The genome assembly completeness as evaluated by benchmarking universal single-copy orthologs was $94.51 \%$ at the eukaryote level. We identified $42.54 \%$ as repeat sequences and a total of 20,536 proteinencoding genes, of which 15,184 genes could be annotated. And we also identified 924 candidate RXLR effectors in the genome assembly. The described genome sequence will provide a valuable resource for better understanding of pathogenicity mechanisms of $P$. vignae and help in uncovering phylogenetical classification of Phytophthora species.
\end{abstract}

\section{Genome Announcement}

Oomycete Phytophthora species are some of the most notorious plant pathogens that cause disease on hundreds of plant species, such as potato late blight disease caused by $P$. infestans, tobacco black shank caused by $P$. nicotianae, and Vigna species stem rot disease caused by $P$. vignae. Soil-borne P. vignae, first identified in 1957 (Purss 1957), produces nonpapillate persistent sporangia and amphigynous antheridia (Stamps et al. 1990). Recent studies suggested that stem and root rot of cowpeas [Vigna unguiculata (L.) Walp. subsp. unguiculata] caused by this homothallic pathogen is emerging as one of the most serious soilborne diseases of cowpea worldwide (Dilantha Fernando and Linderman 1993; Kao and Leu 1982; Kitazawa et al. 1979; Purss 1957; Sivakadadcham and Fernando 1991). It is estimated that $P$. vignae leads to significant losses in cowpea production under favorable environmental conditions (Davis et al. 1994; Purss 1957). Moreover, emergence of new pathogenic races and formae speciales of $P$. vignae possibly contribute to some reported resistant cultivars unavailable in disease management (Kondo et al. 2004; Purss 1957, 1972; Sun et al. 2021). Therefore, in-depth understanding of the molecular mechanisms underlying pathogenicity of $P$. vignae will be conducive to developing sustainable strategies for management of this disease. In this regard, a high-quality complete genome resource for the $P$. vignae strain will be helpful.

${ }^{\dagger}$ Corresponding author: Q. Chen; qhchen@ hainanu.edu.cn

Chengdong Yang and Lianyu Lin contributed equally to this work.

The author(s) declare no conflict of interest.

Accepted for publication 8 March 2021.
(0) PUBLIC The author(s) have dedicated the work to the public domain under the Creative Commons CCO "No Rights Reserved" license by waiving all of his or her rights to the work worldwide under copyright law, including all related and neighboring rights, to the extent allowed by law, 2021. 
Table 1. Genome characteristics

\begin{tabular}{|c|c|}
\hline Features & Strain PSY2020 \\
\hline Oxford Nanopore Technologies (ONT) long reads & $6.83 \mathrm{~Gb}$ (about $76 \times)$ \\
\hline ONT reads $\mathrm{N}_{50}(\mathrm{~kb})$ & 17.05 \\
\hline Illumina short reads & 4.55Gb (about $51 \times$ ) \\
\hline Assembly size (Mb) & 89.39 \\
\hline Contig number & 58 \\
\hline Contig $\mathrm{N}_{50}(\mathrm{Mb})$ & 2.99 \\
\hline Contig $L_{50}$ & 12 \\
\hline Average contig length (Mb) & 1.54 \\
\hline Maximum contig length $(\mathrm{Mb})$ & 6.62 \\
\hline GC content & $55.12 \%$ \\
\hline Repeat sequence & $42.54 \%$ \\
\hline BUSCO assessment in eukaryota $(n=255)^{\mathrm{a}}$ & $94.51 \%$ \\
\hline Protein-encoding genes & 20,536 \\
\hline Candidate RXLR effectors & 924 \\
\hline Genes annotated by Pfam ${ }^{\mathrm{b}}$ & 12,993 \\
\hline Genes annotated by $\mathrm{GO}^{\mathrm{b}}$ & 5,150 \\
\hline Genes annotated by KEGG ${ }^{\mathrm{b}}$ & 8,453 \\
\hline Genes annotated by KOG ${ }^{\mathrm{b}}$ & 14,080 \\
\hline Genes annotated by CAZy ${ }^{b}$ & 463 \\
\hline Total of protein-encoding genes annotated & 15,184 \\
\hline
\end{tabular}

${ }^{a}$ Genome assembly completeness was assessed by benchmarking universal single-copy orthologs (BUSCO).

b Pfam annotation conducted by InterProScan v5.47-82 while Gene Ontology (GO), Kyoto Encyclopedia of Genes and Genomes (KEGG), EuKaryotic Orthologous Groups (KOG), and Carbohydrate Active Enzymes (CAZy) database annotations conducted by EggNogMapper v2.

Here, P. vignae PSY2020 was isolated from an infected cowpea collected from the Hainan Province in China and was incubated on V-8 agar media. High-quality genomic DNA of PSY2020 was extracted and purified, following relative DNA extraction kit protocol, for Oxford Nanopore Technologies (ONT) long reads sequencing and Illumina short reads sequencing. We got $6.83 \mathrm{~Gb}$ of ONT reads, with an $\mathrm{N}_{50}$ read length of $17.05 \mathrm{~Kb}$, and $4.55 \mathrm{~Gb}$ of pairedend Illumina reads, with an insert length of $350 \mathrm{bp}$ (Table 1). The sequencing depth of ONT reads and Illumina reads represent $76 \times$ and $51 \times$ genome coverage, respectively (Table 1 ). To gain genome assembly, we deployed NextDenovo v2.3.1 to generate a draft assembly, using pure ONT reads, and NextPolish v1.3.1 to correct and generate a polished assembly, using both ONT reads and Illumina reads. Finally, we obtained an $89.39-\mathrm{Mb}$ assembly, containing 58 contigs with $2.99 \mathrm{Mb}$ contig $\mathrm{N}_{50}$ and 12 contig $L_{50}$ (Table 1). The average contig length is $1.54 \mathrm{Mb}$ and the largest contig length is $6.62 \mathrm{Mb}$, representing a very high continuous genome assembly of $P$. vignae (Table 1). GC content of PSY2020 is $55.12 \%$, which is very close to isolates of Phytophthora infestans (Ye et al. 2016) (Table 1). The completeness of PSY2020 genome assembly was evaluated through benchmarking universal single-copy orthologs (BUSCO) v4.1.4 (Seppey et al. 2019) with 'eukaryota_odb10' $(n=255)$ as reference dataset. This analysis indicates that genome assembly of strain PSY2020 contains $94.51 \%$ (241 of 255) complete eukaryotic single-copy orthologs (Table 1; Fig. 1B). It is worth noting that proportion of repeat sequence in PSY202 genome is as high as $42.54 \%$, suggesting the rapid amplification of repeat elements in $P$. vignae genome (Table 1; Fig. 1A).

Protein-encoding gene prediction was conducted through a combination of evidencebased prediction by BRAKER2 (Bruna et al. 2021), with $P$. sojae proteins as reference, and $a b$ initio prediction by Fgenesh (Solovyev et al. 2006), with Phytophthora as training organism. A total of 20,536 protein-encoding genes were predicted (Table 1; Fig. 1A). To gain a functional gene annotation, we annotated whole-genome protein-encoding genes with Pfam, GO (Gene Ontology), KEGG (Kyoto Encyclopedia of Genes and Genomes), KOG (EuKaryotic Orthologous Groups), and CAZYmes (carbohydrate active enzymes) databases, respectively. We identified a total of 12,993 proteins with Pfam domains, 5,150 genes with GO items, 8,453 genes involved in different KEGG pathways, 14,080 KOG genes, and 463 CAZYmes (Table 1, Fig. 1A). Overall, we uncovered 1,910 tandem-duplicated genes that generate 797 gene clusters, by exploiting Orthofinder v2.4.1 (Emms and Kelly 2019) (Fig. 1A). To identify more candidate RXLR effectors, we scanned open reading frames containing signal peptide (SignalP v3.0) and RXLR motif but without any transmembrane helices 


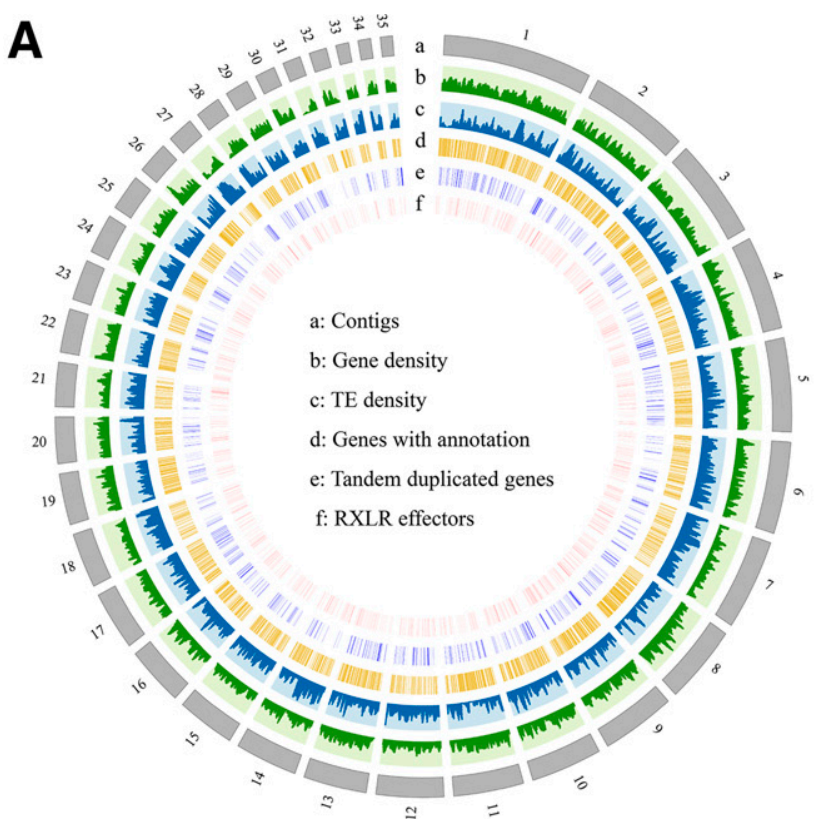

B

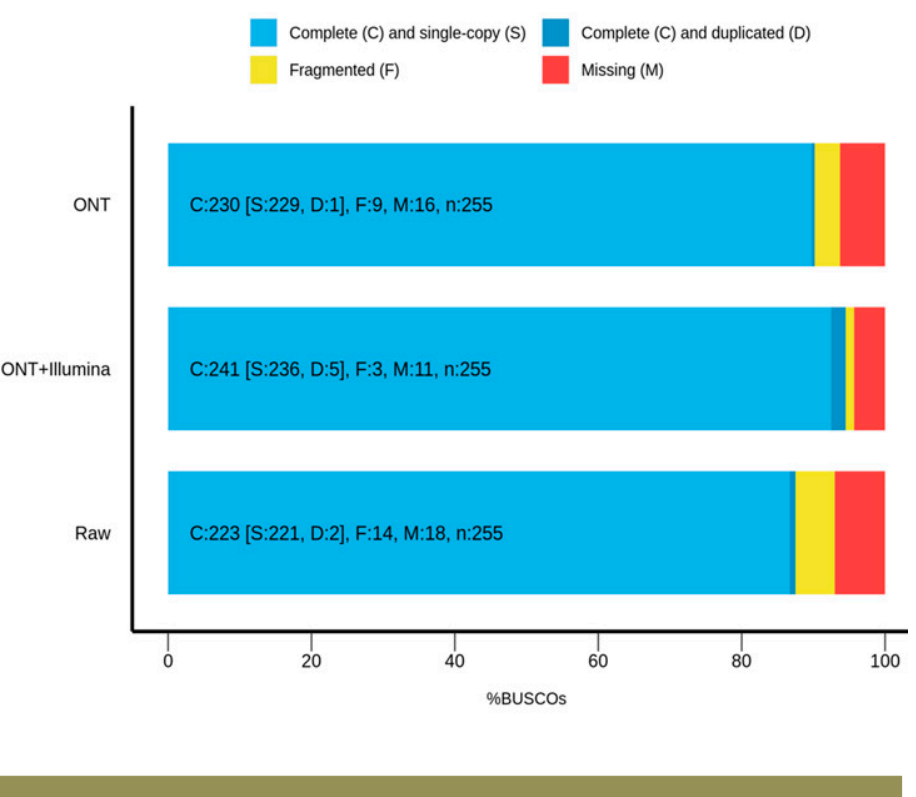

Fig. 1. A, Genome features of Phytophthora vignae PSY2020 were displayed with Circos (Krzywinski et al. 2009). The contigs more than $100 \mathrm{~kb}$ long were displayed in track a. The density of genes and transposable elements (TEs) calculated by sliding window analysis with 100-kb windows and 20-kb steps were shown in tracks b and c, respectively. The locations of genes with functional annotation, tandem duplicated genes, and RXLR effectors were displayed in tracks $d$, e, and f respectively. B, The completeness of genome assembly was assessed by BUSCO v4.1.4 with eukaryotic single-copy orthologs $(n=255)$ from OrthoDB v10. Raw $=$ draft unpolished genome assembly of PSY2020, while ONT = polished assembly corrected with only Oxford Nanopore Technologies (ONT) long reads, and ONT+Illumina $=$ final polished assembly corrected with both ONT long reads and Illumina short reads.

(TMHMM v2.0), following our previous study (PMID 33507115) and, finally, identified 924 candidate RXLR effectors in strain PSY2020 (Table 1; Fig. 1A).

\section{Data Availability}

The whole-genome sequence data reported in this paper have been deposited in the Genome Warehouse in the National Genomics Data Center, Beijing Institute of Genomics (China National Center for Bioinformation), Chinese Academy of Sciences, under accession number GWHAZIG00000000 (BioProject PRJCA004069, BioSample SAMC296216).

\section{Author-Recommended Internet Resources}

Circos: http://circos.ca

Denovo v2.3.1: https://github.com/Nextomics/NextDenovo

Genome Warehouse: http://bigd.big.ac.cn/gwh

NextPolish v1.3.1: https://github.com/Nextomics/NextPolish

\section{Literature Cited}

Bruna, T., Hoff, K. J., Lomsadze, A., Stanke, M., and Borodovsky, M. 2021. BRAKER2: Automatic eukaryotic genome annotation with GeneMark-EP+ and AUGUSTUS supported by a protein database. NAR Genom Bioinform. 1:Iqaa108.

Davis, R. I., Irwin, J. A. G., and Imrie, B. C. 1994. Glasshouse and field evaluation of cowpea lines for partial resistance to Phytophthora vignae. Plant Pathol. 43:17-26.

Dilantha Fernando, W. G. D., and Linderman, R. G. 1993. Occurrence, distribution and pathogenicity of the cowpea root and stem rot pathogen, Phytophthora vignae, in soils of Sri Lanka. Plant Dis. 77:1158-1164.

Emms, D. M., and Kelly, S. 2019. OrthoFinder: Phylogenetic orthology inference for comparative genomics. Genome Biol. 20:238.
Kao, C. W., and Leu, L. S. 1982. Phytophthora stem rot of cowpea caused by Phytophthora vignae Purss in Taiwan. Plant Prot. Bull. 24:189-191.

Kitazawa, K., Suzui, T., and Yanagita, K. 1979. Pathogenicity of Phytophthora vignae Purss to adzuki-bean and cowpea. Ann. Phytopathol. Soc. Jpn. 45:406-408.

Kondo, N., Notsu, A., Naito, S., Fujita, S., and Shimada, H. 2004. Distribution of Phytophthora vignae f.sp. adzukicola races in adzuki bean fields in Hokkaido, Japan. Plant Dis. 88:875-877.

Krzywinski, M., Schein, J., Birol, I., Connors, J., Gascoyne, R., Horsman, D., Jones, S. J., and Marra, M. A. 2009. Circos: An information aesthetic for comparative genomics. Genome Res. 19:1639-1645.

Purss, G. S. 1957. Stem rot: A disease of cowpeas caused by an undescribed species of Phytophthora. J. Agric. Sci. 14:125-154. 
Purss, G. S. 1972. Pathogenic specialisation in Phytophthora vignae. Aust. J Agr. Res. 23:453-456.

Seppey, M., Manni, M., and Zdobnov, E. M. 2019. BUSCO: Assessing genome assembly and annotation completeness. Methods Mol. Biol. 1962:227-245.

Sivakadadcham, B., and Fernando, W. G. D. 1991. Root and stem rot on greenhousegrown cowpea caused by Phytophthora vignae in Sri Lanka. Plant Dis. 75:215.

Stamps, D. J., Waterhouse, G. M., Newhook, F. J., and Hall, G. S. 1990. Revised Tabular Key to the Species of Phytophthora. Mycological Papers. CAB International, Wallingford, U.K.
Solovyev, V., Kosarev, P., Seledsov, I., and Vorobyev, D. 2006. Automatic annotation of eukaryotic genes, pseudogenes and promoters. Genome Biol. 7:S10.11-12.

Sun, F., Sun, S., Yang, Y., Zhou, B., Duan, C., Shan, W., Zhu, Z. 2021. A novel disease of mung bean, Phytophthora stem rot caused by a new forma specialis of Phytophthora vignae. Plant Dis. Published online.

Ye, W., Wang, Y., Shen, D., Li, D., Pu, T., Jiang, Z., Zhang, Z., Zheng, X., Tyler, B. M., and Wang, Y. 2016. Sequencing of the litchi downy blight pathogen reveals it is a Phytophthora species with downy mildew-like characteristics. Mol Plant-Microbe Interact. 29:573-583. 